American J. of Engineering and Applied Sciences 4 (1): 10-16, 2011

ISSN 1941-7020

(C) 2011 Science Publications

\title{
Evaluation of Global Positioning System Data for Offshore Platform Deformation
}

\author{
${ }^{1}$ Abdul Nasir Matori and ${ }^{1,2}$ Nurrohmat Widjajanti \\ ${ }^{1}$ Department of Civil Engineering, University Technology Petronas, Malaysia \\ ${ }^{2}$ Department of Geodesy and Geomatics, Gadjah Mada University, Indonesia
}

\begin{abstract}
Problem statement: Reservoir compaction and shallow gas migration phenomena may cause offshore platform to experience deformation which if happens excessively will affect their structural integrity. Approach: Hence it is crucial to monitor and quantify the magnitude of the deformation especially if they are not uniform throughout the platform structure. However since mostly the offshore platforms are few hundreds kilometers away from shore, the precise monitoring of their deformation is limited to very few sophisticated instruments, in which GPS technology is one of them albeit using very special GPS data processing technique such as Long Baseline Relative Positioning. Results: Using this technique and employing GPS data observed on one of PETRONAS own platform, Pulai, its deformation magnitude will be determined with various options such as number of reference stations used, configuration and their geographic location. This study presents initial deformation processing result using scientific software GAMIT/GLOBK and their analysis utilizing postfit nrms and chi-squared statistics. The result indicated that for the period of two months there was displacement as big as $0.0094 \mathrm{~m}$ with standard deviation of $0.0106 \mathrm{~m}$. However following congruency statistical test using t-student distribution with $95 \%$ confidence level, indicated that this displacement is insignificance. Analysis of the output result with postfit nrms also indicated that the data were of good quality, the processing procedure was correct and the output for each processing epoch is internally and externally consistent. Conclusion/Recommendations: It could be concluded with correct data processing strategy GPS data could be used to determine deformation magnitude which consequently could be utilized as input to assess structural integrity of an offshore platform.
\end{abstract}

Keywords: GPS data, offshore platform, deformation, global positioning system

\section{INTRODUCTION}

One of the major problems of engineering structure such as platform is the occurrence of deformation, which may cause disaster to human being and platform itself. Applying a deformation monitoring system to monitor this stability is a rational approach to address the safety of the platform. Because of its remote location, deformation of offshore platform needs to be observed by using GPS technique. GPS offers several advantages over other types of technology. It requires minimal user interaction and provides 3-dimensional position information. Furthermore the other benefit is possibility of acquisition of large number of high resolution observables at relatively high speed in any weather condition.

In this s, Long baseline Relative Positioning using continuous GPS data is being investigated for the early detection of a platform deformation. The research of the capabilities of GPS positioning for the continuous and accurate monitoring of platform has been carried out in
Malaysian water (Setan et al., 2004). Pulai platforms is located in South China Sea and about $300 \mathrm{~km}$ from the shore of east Peninsular Malaysia was observed using GPS method to detect its deformation. GPS data processing in this case requires sophisticated modeling and processing strategies in order to find the deformation which is not contaminated by error or bias. Therefore the GPS data processing will utilize highly sophisticated scientific software GAMIT/GLOBK to address high precision requirement of deformation monitoring. Preliminary deformation analysis using GPS data observed on Pulai Platform in 2003 indicated there was no significant deformation detected as mentioned in (Nurrohmat and Matori, 2008).

Relative positioning GPS: The fundamental concept of unstable object monitoring is to apply GPS relative positioning with reference to the more stable control station. A number of control stations between reference station (i.e., control points on shore) and object station (i.e., control points on offshore platforms) need to be

Corresponding Author: Nurrohmat Widjajanti, Department of Civil Engineering, University Technology Petronas, Malaysia 


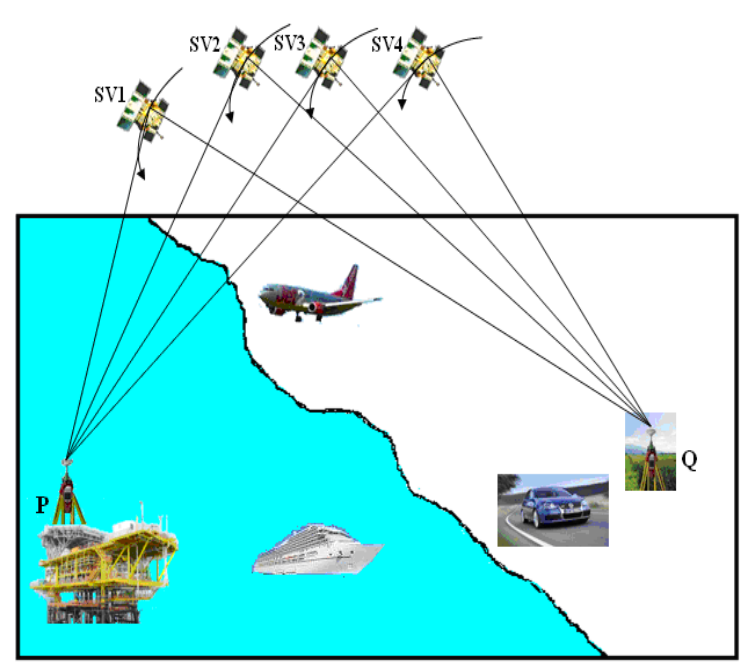

Fig. 1: Relative positioning using GPS technique

set up for measurement in the form of baseline vectors $(\Delta \mathrm{x}, \Delta \mathrm{y}, \Delta \mathrm{z})$. This is to reach a high precision that is essential for deformation monitoring (Leick, 2004; Setan and Othman, 2006).

The relative positioning technique (Fig. 1) involves control point on shore (station Q) and control point set up at the offshore platform (station $\mathrm{P}$ ). The relative static GPS observations are carried out for 24 hours to provide sufficient required precision for a long-baseline positioning. Relative positioning requires at least two receivers, one as the reference and the other as the object station (Hofmann-Wellenhof et al., 2001). Both receivers observe the same satellite to collect the observation data at both stations then used to estimate the position of the object station, relative to the reference station.

The technique of relative positioning could reduce and remove most of the biases common to the receiver through the use of the doubly-differenced algorithm (Popovas and Radzeviciute, 2001) hence could provide result with high precision.

\section{MATERIALS AND METHODS}

The following section will explain the utilization of relative positioning to quantify deformation together with the data and relevant software or tools used.

GAMIT/GLOBK software: GAMIT is a series of programs that analyze GPS phase data to estimate 3dimensional relative positions of ground stations and satellite orbital parameters, earth orientation parameter and atmospheric delay parameters (Dong et al., 1998). GAMIT is categorized as scientific software tends which have been developed by Massachusetts Institute of Technology, USA for research and precise positioning purposes. This software has the capability to process more than one observation simultaneously.

GAMIT generates the answer with only loose constraints on the parameters. In order to avoid several bias of the combination, it hence requires last GLOBK step to identify the reference frame by imposing constraints on stations' coordinates (Dong et al., 1998). GLOBK is a Kalman filter process for combining solutions of the primary data processing from space-geodetic or terrestrial observations.

GLOBK accepts the estimated data and associated covariance matrices for station coordinates and other parameter generated from analyses of the primary observations. These main solutions should be performed with loose a priori uncertainties assigned to the global parameters, so that the constraints can be applied uniformly in the combined solution (Dong et al., 1998).

GPS Data Measurement: The GPS data observed in three epochs using Trimble 4000 SSI Geodetic series GPS receiver. Each session was planned as a period of 24 hours continuously on a daily basis. The recording interval was $15 \mathrm{sec}$ with at least $10^{\circ}$ mask-angles configuration. Surveys campaign was carried out by Fugro Geodetic (Malaysia) Sdn Bhd.

Processing utilized eight days GPS (rinex) data from epoch 1 to epoch 3. Figure 2 shows the schematic scenario of the computation.

The network configuration is illustrated in Fig. 3 with Pulai offshore platform as object station and Malaysian primary GPS as regional reference stations. These Malaysian primary stations are BESE (BeserahKuantan), TERE (Kuala Terengganu) and PANJ (Rantau Panjang). The processing also applied global reference station from International GPS Services (IGS) which are PIMO (Quezon City-Philippines), $\mathrm{COCO}$ (Cocos Keeling-Australia) and BAN2 (Bangalore-India). The IGS rinex GPS and precise ephemeris data were downloaded from IGS (International GNNS services) website. The sketch of IGS stations location could be seen in Fig. 4.

GPS DataProcessing: The processing of the GPS data used the GAMIT software to generate daily solution. Then, these solutions were combined in order to find station coordinates in the reference frame using GLOBK software (Dong et al., 1998).

Prior to the above steps there were other softwares required to assist the data processing which are: 1 ) 


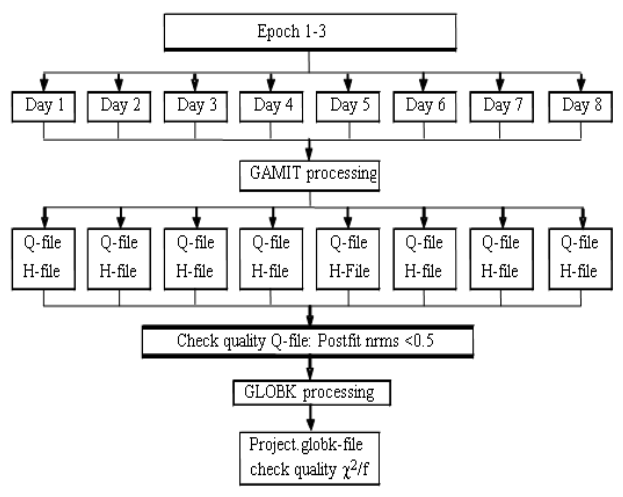

Fig. 2: The flowchart of Pulai platform deformation computation

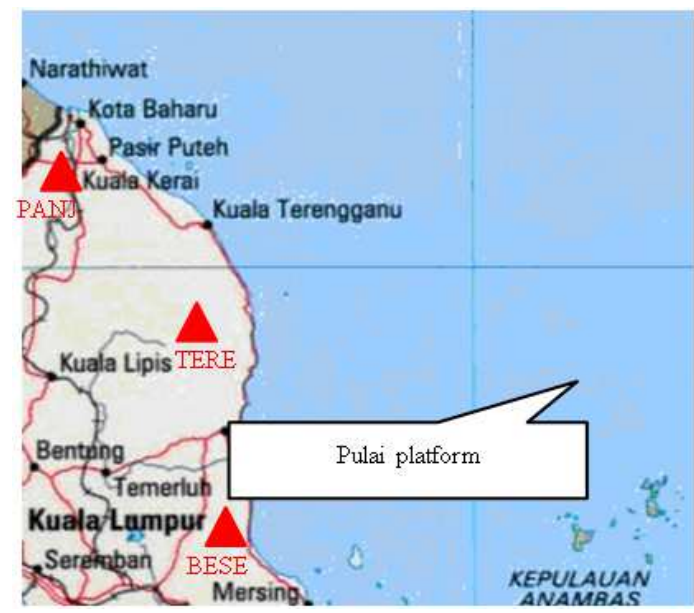

Fig. 3: Malaysian primary GPS stations location

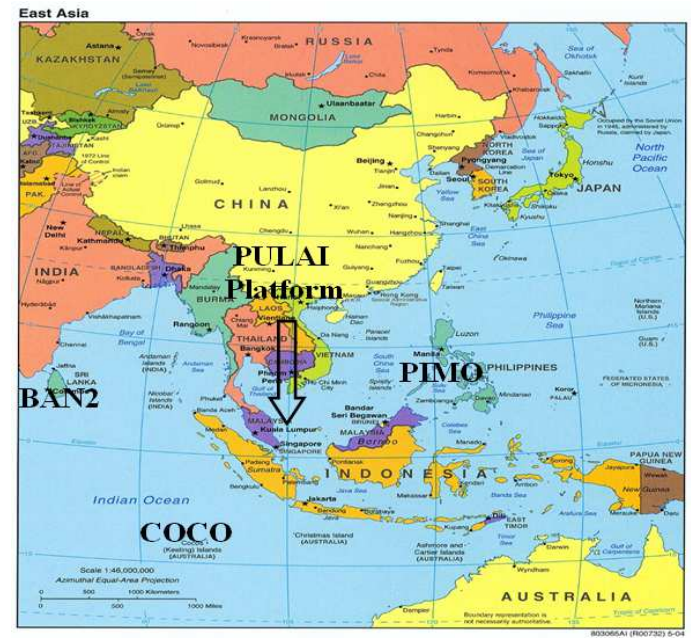

Fig. 4: Sketch of global station from IGS
Fedora Core with Linux Operating System, 2) TEQC software to organize three main functions namely Translating, Editing and Quality Checking of rinex file, 3) NetCDF (Network Common Data Form) interface for sharing and transfering access of scientific data especially in interface between C, Fortran-77 and Fortran-90 for GAMIT installation, 4) GMT (Generic Mapping Tools) for data plotting of GAMIT output.

\section{RESULTS}

Primary indicator of GAMIT and GLOBK solution: The next explanation is the preliminary result of GAMIT/GLOBK processing. The number of stations at each daily processing are not the same in every epoch. For example, there are GPO1, GPO2, GPO3, Malaysian primary station and IGS station for first day and GPO4, GPO5, GPO6, Malaysian primary station and IGS station for the seventh day. On the other hand, there are GPO1, GPO2, GPO3, GPO4, GPO5, GPO6, Malaysian primary station and IGS station for the sixth day. The scenario of data processing in each day depends on the available GPS data.

The primary indicator of GAMIT solution for determining the acceptable solution is the normalized root mean square (nrms) i.e. the square root of chisquare per degree of freedom. Usually with the default weighting scheme, a good solution produce an nrms about 0.25 (Dong et al., 1998). Figure 5a shows the nrms of the daily GAMIT solution from the Q-file output. The result indicates the nrms of about 0.2 and the nrms value for each day processing is very close. It shows the data used are those with high quality where the errors are randomly distributed and the correct station apriori weight used. Furthermore there are no serious modeling problems apparent since the nrms is relatively small and less than 0.25 which is considered to be a good high ionosphere and solution procedures. The graph in Fig. 5b depicts mean and standard deviation of postfit nrms in each epoch. The postfit nrms mean of epoch 2 and 3 has the same value about 0.20 , while epoch 1 is equal to 0.15 . Fig. $5 \mathrm{~b}$ also present standard deviation value of epoch 2 and 3 equal to 0.01 . The standard deviation is smaller than the value of epoch 1 .

The primary quality assurance statistic of GLOBK solution is $\chi^{2} / \mathrm{f}$, the chi-squared increment per degree of freedom. Table 1 shows the $\chi^{2} / \mathrm{f}$ statistic of daily and global processing from GLOBK output. The global processing is run by combination of all daily solutions. Shown in this table, the individual values never exceeded 3.0, indicating that each of the campaigns is internally and externally consistent (Lestari, 2006). 
Am. J. Engg. \& Applied Sci., 4 (1): 10-16, 2011

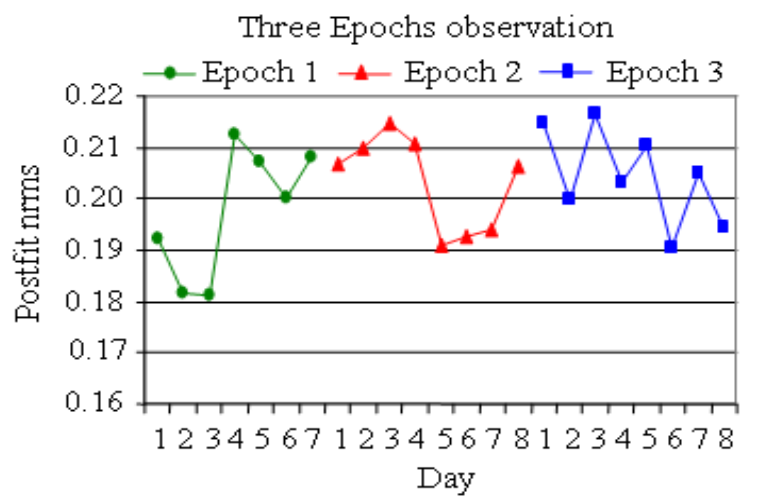

(a)

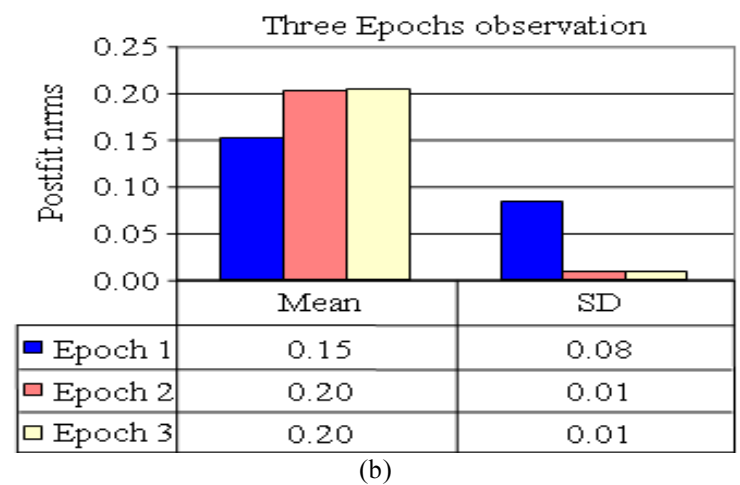

Fig. 5: Primary quality of daily GAMIT processing. (a) Postfit nrms; (b) mean and standard deviation

Table 1: $\chi^{2} /$ f statistic of daily and global processing from GLOBK output

\begin{tabular}{|c|c|c|c|c|c|c|c|c|c|}
\hline & \multicolumn{8}{|l|}{ Day } & \multirow[b]{2}{*}{ Global } \\
\hline & 1 & 2 & 3 & 4 & 5 & 6 & 7 & 8 & \\
\hline \multicolumn{10}{|c|}{ Epoch 1} \\
\hline $\begin{array}{l}\chi^{2} / f \\
\text { Epocl }\end{array}$ & $\begin{array}{l}0.645 \\
2\end{array}$ & 0.914 & 0.727 & 0.502 & 0.511 & 0.449 & 0.468 & & 0.654 \\
\hline $\begin{array}{l}\chi^{2} / f \\
\text { Epocl }\end{array}$ & $\begin{array}{l}0.559 \\
2\end{array}$ & 0.433 & 0.460 & 0.393 & 0.151 & 0.356 & 0.469 & 0.739 & 0.566 \\
\hline$\chi^{2} / \mathrm{f}$ & 0.564 & 0.461 & 0.446 & 0.463 & 0.571 & 0.597 & 0.277 & 0.725 & 0.564 \\
\hline
\end{tabular}

Estimated Coordinate and Its Precision: The GAMIT $\mathrm{H}$-file output associated with each day in the campaign is combined together using GLOBK. One of GLOBK result is coordinate in local system with horizontal component i.e., North (N) and East (E) and vertical component i.e., Up (U) and also their standard deviation. The N, E and $U$ are products of adjustment in Geodetic latitude, longitude and height respectively.

Table 2 shows the N, E and U estimated coordinate in meters of the six object stations for three epochs processing. As shown in the table, there is a little bit variation in the third $\mathrm{N}, \mathrm{E}$ and $\mathrm{U}$ coordinates.
Table 2: Estimated coordinate in local system

\begin{tabular}{|c|c|c|c|}
\hline \multirow[b]{2}{*}{ Station } & \multicolumn{3}{|l|}{ Coordinate } \\
\hline & Epoch 1 & Epoch 2 & Epoch 3 \\
\hline \multicolumn{4}{|l|}{$\overline{\text { GPO1 }}$} \\
\hline North (m) & 593207.89252 & 593207.89048 & 593207.88819 \\
\hline East (m) & 11692467.77186 & 11692467.77280 & 11692467.77482 \\
\hline $\mathrm{Up}(\mathrm{m})$ & 38.34215 & 38.33709 & 38.33438 \\
\hline \multicolumn{4}{|l|}{ GPO2 } \\
\hline North (m) & 593181.00532 & 593181.00329 & 593181.00195 \\
\hline East (m) & 11692463.02954 & 11692463.03050 & 11692463.03187 \\
\hline $\mathrm{Up}(\mathrm{m})$ & 38.21905 & 38.21389 & 38.21082 \\
\hline \multicolumn{4}{|l|}{ GPO3 } \\
\hline North (m) & 593223.53160 & 593223.52963 & 593223.52733 \\
\hline East (m) & 11692414.83799 & 11692414.83895 & 11692414.83699 \\
\hline $\mathrm{Up}(\mathrm{m})$ & 38.86909 & 38.86396 & 38.86540 \\
\hline \multicolumn{4}{|l|}{ GPO4 } \\
\hline North (m) & 593494.33695 & 593494.33501 & 593494.33392 \\
\hline East (m) & 11691033.57984 & 11691033.58071 & 11691033.58165 \\
\hline $\mathrm{Up}(\mathrm{m})$ & 31.65561 & 31.65036 & 31.65313 \\
\hline \multicolumn{4}{|l|}{ GPO5 } \\
\hline North (m) & 593481.51835 & 593481.51627 & 593481.51394 \\
\hline East (m) & 11691021.59712 & 11691021.59806 & 11691021.59318 \\
\hline $\mathrm{Up}(\mathrm{m})$ & 31.69202 & 31.68752 & 31.68546 \\
\hline \multicolumn{4}{|l|}{ GPO6 } \\
\hline North (m) & 593510.39973 & 593510.39778 & 593481.40170 \\
\hline East (m) & 11690987.08537 & 11690987.08630 & 11690987.08834 \\
\hline $\mathrm{Up}(\mathrm{m})$ & 27.93700 & 27.93186 & 27.93464 \\
\hline
\end{tabular}

Figure 6a-c shows the precision of N, E and $U$ in a series. Figure 6a-c indicate there are a slightly difference in standard deviation between processing of first, second and third epoch. The horizontal component ( $\mathrm{N}$ and $\mathrm{E}$ ) have similar precision of 5-6 $\mathrm{mm}$.

The vertical component (U) traditionally has poorer precision than the horizontal component with its maximum value here is approximately $8 \mathrm{~mm}$. The precision of six stations of the three epochs processing are similar for horizontal and vertical component.

Fig. 8a illustrates sketch of horizontal movement of point GOP1, GOP2 and GOP3, while Fig. 8b presents sketch of point GOP4, GOP5 and GOP6. The movement is represented by magnitude and direction between epochs 1-2, epochs 2-3 and epochs 2-3.

Displacement Test: As aforementioned, the result yields different coordinate between two epochs, the difference then is called as movement. As can be seen in Fig. $7 \mathrm{a}$ and $\mathrm{b}$, there are horizontal and vertical movement between each two epochs. The horizontal movement is shown by magnitude and direction. The magnitude is value of these movements while the direction is azimuth from a station in first epoch to the station in second epoch at the same station. Fig. 7a shows that the magnitude of coordinate difference is generally in level of a few millimetres. A different direction is found in the horizontal movement between two epochs. The vertical movement is shown in Fig 7b. The biggest and smallest value of the movement is 
Am. J. Engg. \& Applied Sci., 4 (1): 10-16, 2011

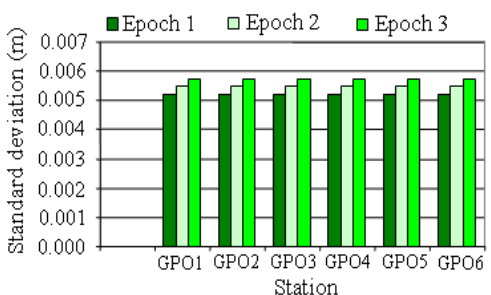

(a)

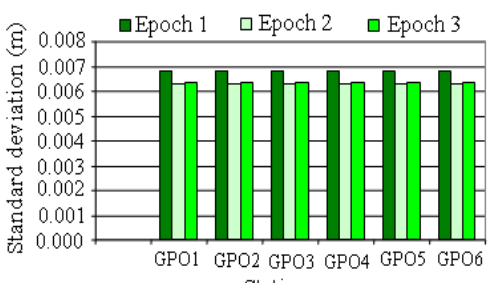

(b)

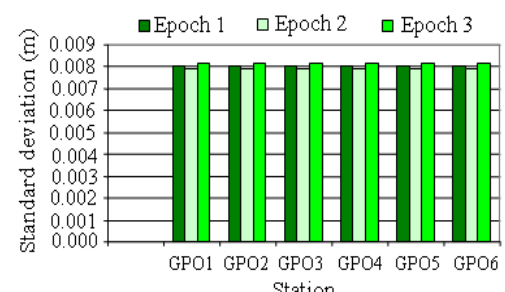

(c)

Fig. 6: Standard deviation of N, E and U coordinates. (a) precision of North; (b) precision of East; (c) precision of Up

\begin{tabular}{|c|c|c|c|c|c|c|}
\hline \multirow{2}{*}{ Station } & \multicolumn{2}{|c|}{ Epoch1-Epoch2 } & \multicolumn{2}{c|}{ Epoch2-Epoch3 } & \multicolumn{2}{c|}{ Epoch1-Epoch3 } \\
\cline { 2 - 7 } & Magnitude (m) & Direction & Magnitude (m) & Direction & Magnitude (m) & Direction \\
\hline \hline GOP1 & 0.0022 & $131026^{\prime} 5.57^{\prime \prime}$ & 0.0031 & $114042^{\prime} 24.6^{\prime \prime}$ & 0.0052 & $124021^{\prime} 11.77^{\prime}$ \\
\hline GOP2 & 0.0022 & $135039^{\prime} 7.51^{\prime \prime}$ & 0.0019 & $115024^{\prime} 42.4^{\prime \prime}$ & 0.0041 & $124042^{\prime} 54.7^{\prime \prime}$ \\
\hline GOP3 & 0.0022 & $229032^{\prime} 35.8^{\prime \prime}$ & 0.0030 & $115052^{\prime} 28.7^{\prime \prime}$ & 0.0044 & $256045^{\prime} 4.17^{\prime \prime}$ \\
\hline GOP4 & 0.0021 & $130047^{\prime} 37.7^{\prime \prime}$ & 0.0014 & $11404^{\prime} 22.12^{\prime \prime}$ & 0.0035 & $120048^{\prime} 51.6^{\prime \prime}$ \\
\hline GOP5 & 0.0023 & $205030^{\prime} 44.2^{\prime \prime}$ & 0.0054 & $114024^{\prime} 45.1^{\prime \prime}$ & 0.0059 & $228011^{\prime} 49.4^{\prime \prime}$ \\
\hline GOP6 & 0.0022 & $62028^{\prime} 55.3^{\prime \prime}$ & 0.0044 & $115029^{\prime} 40.8^{\prime \prime}$ & 0.0036 & $33031^{\prime} 12.09^{\prime \prime}$ \\
\hline
\end{tabular}

(a)

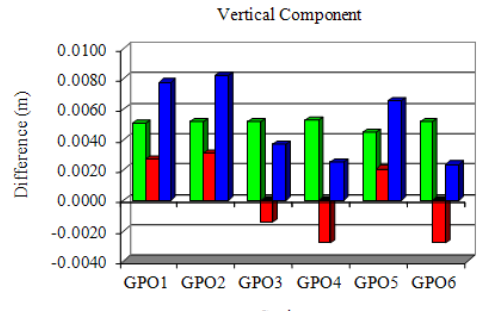

口Epoch1-Epoch2

appoch2-Epoch3

Epoch1-Epoch3

(b)

Fig. 7: Vertical and horizontal difference between two epochs. (a) horizontal component; (b) vertical component

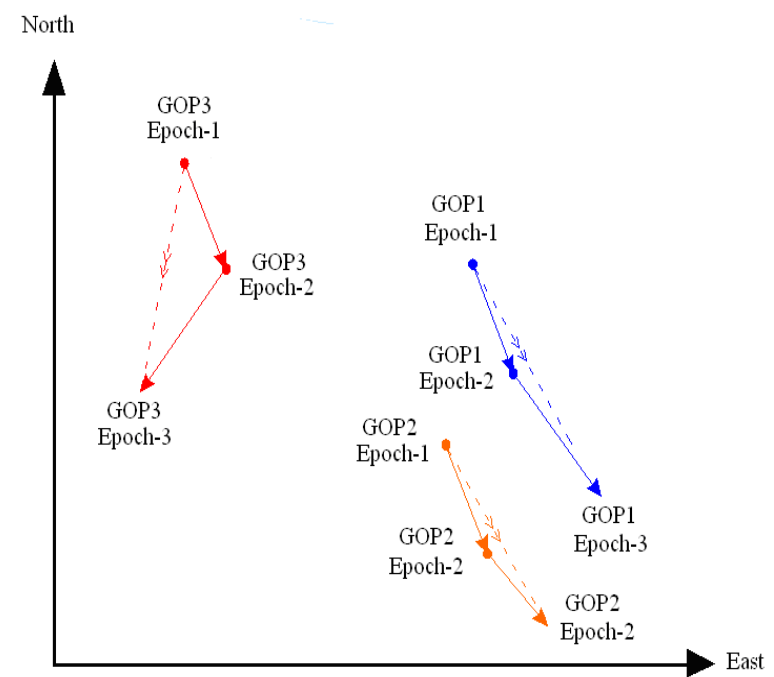

(a)

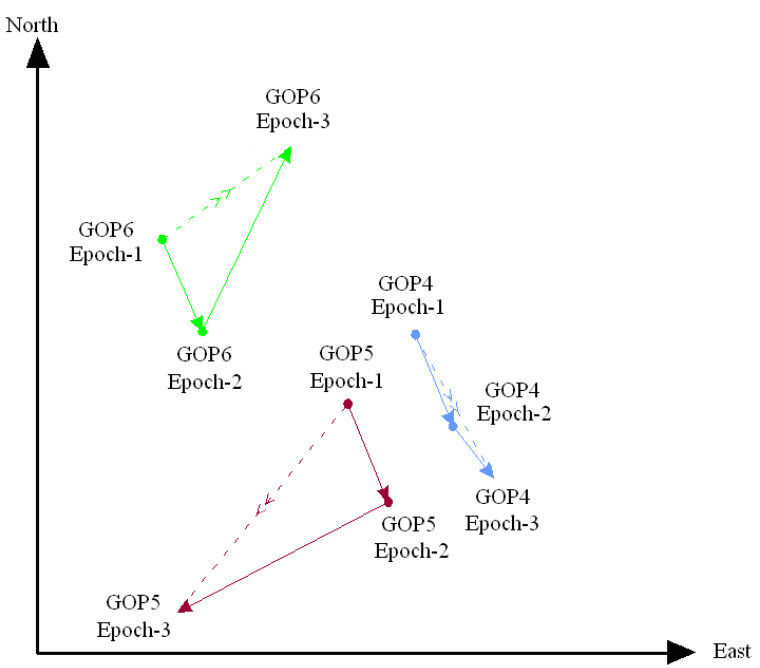

(b)

Fig. 8: Sketch of horizontal movement. (a) point GOP1, GOP2 and GOP3; (b) point GOP4, GOP5 and GOP6

$0.0082 \mathrm{~mm}$ at point GOP2 (between epoch 1-3) and $0.0014 \mathrm{~mm}$ at point GOP3 (between epoch 2-3). The almost movement have positive value. It is noted that the value of first epoch is bigger than second epoch.

The displacement of a station between epoch $\mathrm{i}$ and $\mathrm{j}\left(\Delta \mathrm{d}_{\mathrm{ij}}\right)$ is shown in following Eq. 1 (Kuang, 1996):

$$
\Delta \mathrm{d}_{\mathrm{ij}}=\left(\Delta \mathrm{E}_{\mathrm{ij}}^{2}+\Delta \mathrm{N}_{\mathrm{ij}}^{2}+\Delta \mathrm{U}_{\mathrm{ij}}^{2}\right)^{0.5}
$$

Where $\Delta \mathrm{E}, \Delta \mathrm{N}$ and $\Delta \mathrm{U}$ are the coordinate differences of $\mathrm{E}, \mathrm{N}$ and $\mathrm{U}$ between both epochs respectively. 
Table3. Displacement, its standard deviation and T-computed

\begin{tabular}{|c|c|c|c|}
\hline \multirow[b]{2}{*}{ Station } & \multicolumn{3}{|c|}{ Displacements } \\
\hline & $\Delta \mathrm{d}_{12}(\mathrm{~m})$ & $\sigma_{(\Delta 12)}(\mathrm{m})$ & $\mathrm{T}$ \\
\hline \multicolumn{4}{|c|}{ (a) between epoch 1 and 2} \\
\hline GOP1 & 0.0055 & 0.0108 & 0.513 \\
\hline GOP2 & 0.0056 & 0.0108 & 0.521 \\
\hline GOP3 & 0.0056 & 0.0108 & 0.515 \\
\hline GOP4 & 0.0057 & 0.0109 & 0.521 \\
\hline GPO5 & 0.0050 & 0.0107 & 0.473 \\
\hline \multirow[t]{2}{*}{ GOP6 } & 0.0056 & 0.0108 & 0.515 \\
\hline & $\Delta \mathrm{d}_{23}(\mathrm{~m})$ & $\sigma_{(\Delta 23)}(\mathrm{m})$ & \\
\hline \multicolumn{4}{|c|}{ (b) between epoch 2 and 3} \\
\hline GOP1 & 0.0041 & 0.0098 & 0.416 \\
\hline GOP2 & 0.0036 & 0.0106 & 0.341 \\
\hline GOP3 & 0.0033 & 0.0090 & 0.372 \\
\hline GOP4 & 0.0031 & 0.0108 & 0.289 \\
\hline GOP5 & 0.0058 & 0.0091 & 0.634 \\
\hline \multirow[t]{2}{*}{ GOP6 } & 0.0052 & 0.0092 & 0.569 \\
\hline & $\Delta \mathrm{d}_{13}(\mathrm{~m})$ & $\sigma_{(\Delta 13)}(\mathrm{m})$ & \\
\hline \multicolumn{4}{|c|}{ (c) between epoch 1 and 3} \\
\hline GOP1 & 0.0094 & 0.0106 & 0.887 \\
\hline GOP2 & 0.0092 & 0.0109 & 0.842 \\
\hline GOP3 & 0.0057 & 0.0095 & 0.603 \\
\hline GOP4 & 0.0043 & 0.0094 & 0.459 \\
\hline GOP5 & 0.0088 & 0.0102 & 0.863 \\
\hline GOP6 & 0.0043 & 0.0097 & 0.439 \\
\hline
\end{tabular}

To prove that the displacement is significant, congruency statistical test is adopted in this study using tstudent distribution as suggested by (Caspary, 1987). The null hypothesis of the test is $\Delta \mathrm{d}_{\mathrm{ij}}=0$, that there is no movement between two epochs. The number of Tcomputed is the ratio of the displacement and its standard deviation $\left(\mathrm{S}_{(\Delta \mathrm{dij})}\right)$. This standard deviation is determined by error propagation approach from the standard deviation of coordinates.

The $\mathrm{T}$ statistics value for the test can be expressed as Eq. (2):

$\mathrm{T}=\Delta \mathrm{d}_{\mathrm{ij}} / \sigma_{(\Delta \mathrm{dij})}$

The region where the null hypothesis is accepted is $\mathrm{T}<\mathrm{t}_{\mathrm{f}, \alpha / 2}$ (Wolf and Ghilani, 1997). The critical value of $\mathrm{t}$ is found in the t-table corresponding to the significance level $(\alpha)$ and the appropriate degrees of freedom (f). In this study, degree of freedom is $\infty$ because of the GPS data was recorded in 24 hours observation with $15 \mathrm{sec}$ data interval. By using confidence level of $95 \%(\alpha=$ $5 \%)$ then t-value $(\infty, 0.025)$ is 1.960 (Wolf and Ghilani, 1997). Table 3 illustrates the value of displacement, its standard deviation and also T-computed for six stations

Table $3 \mathrm{a}$ depicts the result of $\mathrm{t}$-test of displacement between epoch 1 and 2, Table $3 b$ is result between epoch 2 and 3 and Table $3 \mathrm{c}$ shows $\mathrm{T}$ value between epoch 1 and 3 . From these table $(3 a, b, c)$, it can be identified that the $\mathrm{T}$ value is smaller than 1.960 . It means that significant displacement was not found in all stations in the three observation epochs by implementing the congruency test. It could be statistically declared that with $95 \%$ confidence level, there was no deformation of Pulai platform as observed in the three epochs.

\section{DISCUSSION}

Analysis of GPS data-derived coordinates of the monitored points on the observed platform (Pulai) with postfit nrms indicated that the data used were of good quality and the procedure applied in the process were correct. Furthermore using $\chi^{2} / f$ to check the quality of the output of GLOBK processing it showed that each epoch was internally and externally consistent.

Based on the GPS data processing result, it could be summarized that there were coordinate differences within the three epoch observations. Nevertheless, these differences were not statistically categorized as a significant displacement as a result of congruence test using t-student distribution.

\section{CONCLUSION}

From the outcome of this study it was proven the GPS relative positioning technique is a reliable method for deformation monitoring of an offshore platform. This is due to its capability of detecting subsidence magnitude in few centimeters, or even several millimeters level. However in order to obtain that level of precision is not a simple job to perform. It this case, the GPS technique should be implemented with some appropriate consideration which begins with choosing a dual frequency geodetic type GPS receiver and applying a particular strategy in both observation (duration, mask-angle, recording interval) and processing such as quality data checking, error and bias removal, number and configuration of reference stations to be used as well as resolving any ambiguity resolution. These several options were used as an input parameter in determining strategy of processing in scientific software such as GAMIT/GLOBK.

It is also highlighted that for such high precision positioning purposes such as the above mm level subsidence detection the utilizing of high performance software such as GAMIT/GLOBK for processing GPS data is recommended. This study has shown also that offshore platform subsidence could be monitored as an effort to increase their safety and life-time. 


\section{ACKNOWLEDGMENT}

We would like to thank Petronas Carigali Sdn Bhd and Fugro Geodetic (Malaysia) Sdn Bhd for providing GPS data used in this study.

\section{REFERENCE}

Caspary, W.F., 1987. Concepts of Network and Deformation Analysis. 2nd Edn., School of Surveying, The University of New South Wales, Kensington, ISBN-10: 0858390442, pp: 183.

Dong, D., T.A. Herring and R.W. King, 1998. Estimating regional deformation from a combination of space and terrestrial geodetic data. J. Geodesy, 72: 200-214. DOI: $10.1007 / \mathrm{s} 001900050161$

Hofmann-Wellenhof, B., H. Lichtenegger and J. Collins, 2001. Global Positioning System: Theory and Practice. 5th Edn., Springer-Verlag, New York, ISBN-10: 3211835342, pp: 382.

Kuang, S., 1996. Geodetic Network Analysis and Optimal Design: Concepts and Applications. 1st Edn., Ann Arbor Press, USA., ISBN: 1575040441, pp: 368.
Leick, A., 2004. GPS Satellite Surveying. 3rd Edn., John Wiley and Sons, USA., ISBN: 0471059307, pp: 435.

Lestari, D., 2006. GPS Study for Resolving the Stability of Borobudur Temple Site. 1st Edn., The University of New South Wales, Sydney, Australia, pp: 312 .

Popovas, D. and K. Radzeviciute, 2001. Adjustment of the Lithuanian GPS Network Using GAMIT. 1st Edn., Aalborg University, Institute of Development and Planning, Denmark, pp: 190.

Setan, H. and R. Othman, 2006. Monitoring of offshore platform subsidence using permanent GPS stations. J. Global Position. Sys., 5: 17-21.

Setan, H., M.S. Ibrahim, A.N. Matori and S.M. Saat, 2004. Initial investigation of land subsidence using GPS data and rigorous analysis. Proceedings of the International Symposium on GNSS/GPS, Dec. 6-8, GNSS, Sydney, Australia, pp: 1-12.

Wolf, P.R. and C.D. Ghilani, 1997. Adjustment Computations: Statistics and Least Squares in Surveying and GIS. 3rd Edn., John Wiley and Sons, USA., ISBN-10: 0471168335 pp: 564. 